\title{
BMJ Open Protocol for a systematic review and meta- analysis of the placebo response in treatment-resistant depression: comparison of multiple treatment modalities
}

\author{
Brett D M Jones (D , 1,2 Cory R Weissman, ${ }^{1,2}$ Lais B Razza, ${ }^{3,4}$ M Ishrat Husain, ${ }^{1,2}$ \\ Andre R Brunoni, ${ }^{3,4}$ Zafiris J Daskalakis ${ }^{5}$
}

To cite: Jones BDM, Weissman CR, Razza LB, et al. Protocol for a systematic review and meta-analysis of the placebo response in treatment-resistant depression: comparison of multiple treatment modalities. BMJ Open 2021;11:e041349. doi:10.1136/ bmjopen-2020-041349

- Prepublication history for this paper is available online. To view these files, please visit the journal online (http://dx.doi. org/10.1136/bmjopen-2020041349).

Received 05 June 2020 Revised 09 December 2020 Accepted 18 December 2020

Check for updates

(C) Author(s) (or their employer(s)) 2021. Re-use permitted under CC BY-NC. No commercial re-use. See rights and permissions. Published by BMJ.

For numbered affiliations see end of article.

Correspondence to Dr Zafiris J Daskalakis; zdaskalakis@health.ucsd.edu

\section{ABSTRACT}

Introduction The high placebo response in depression treatment trials is a major contributing factor for randomised control trial failure to establish efficacy of novel or repurposed treatments in treatment-resistant depression (TRD) and major depressive disorder in general. Though there have been a number of meta-analyses and primary research studies evaluating the placebo response in non-TRD, placebo response in TRD is poorly understood. It is important to understand the placebo response of TRD as treatments are only moderately effective and up to $1 / 3$ of patients will experience TRD.

Methods and analysis We will conduct a search of electronic databases (MEDLINE and PsychINF0) from inception to 24th January 2020 including randomised, placebo-controlled trials of pharmacological, somatic and psychological interventions for adults with TRD. TRD will be defined as a failure to respond to at least two interventions of adequate dose or duration. We will also search reference lists from review articles. We will perform several meta-analyses to quantify the placebo response for each treatment modality. Regression analysis will explore potential contributing demographic and clinical variables to the placebo response. We will use Cochrane risk of bias tool.

Ethics and dissemination There is no research ethics board approval required. The dissemination plan is to publish results in a peer-reviewed academic journal. PROSPERO registration number 190465.

\section{INTRODUCTION}

The placebo response is the therapeutic effect produced by a placebo intervention that is not due to any inherent properties of the placebo itself. The high placebo response in large depression treatment trials is a major contributing factor for randomised control trial (RCT) failure to establish efficacy of novel and repurposed treatments. ${ }^{1}$ There have been several studies attempting to determine patient and study variables contributing to the placebo response in non-treatmentresistant depression (TRD). Variables found to contribute to the placebo response include
Strengths and limitations of this study

- This analysis will be the first to exclusively study the placebo response in treatment-resistant depression.

- We will seek to quantify the placebo response among distinct treatment modalities.

- The study will assess a large number of variables that may contribute to the placebo response.

- Direct statistical comparison between the placebo response of treatment modalities will not be done given the significant heterogeneity among treatment modalities.

year of publication, baseline severity, probability of being allocated to placebo arm, number of clinic centres, dosing schedule, length of trial, the magnitude of active response, early score fluctuations and inflation of baseline severity. ${ }^{2-10}$ The largest metaanalysis to date (252 studies, pooled $\mathrm{n}=26324$ ) reported that the placebo response rate of antidepressant medications has been stable over the last thirty years and ranges between $35 \%$ and $40 \% .{ }^{11}$ While the placebo response is extensively investigated in non-TRD, there is a paucity of research into the magnitude of the placebo response in TRD.

TRD is defined by a lack of response to at least two separate treatments and imposes a heavy burden on the individual, their families and society, through decreased quality of life, increased morbidity and direct/indirect medical costs. ${ }^{12}{ }^{13}$ It is important to integrate novel treatments into clinical practice; however, a high placebo response and negative clinical trials have led to a delay in this regard. To address this gap, it is important to characterise and understand the placebo response in TRD. Two meta-analyses have explored the placebo response in repetitive transcranial magnetic stimulation trials, including patients with TRD and non-TRD, 
which reported a large placebo response. ${ }^{14}{ }^{15}$ To date, these are the only studies attempting to characterise the placebo response in TRD.

Currently, there is not a clear understanding as to what the placebo response in TRD is, what contributes to it and how it may differ across various treatment modalities. Hence, we will complete a systematic review and meta-analysis of randomised, placebo-controlled trials in TRD. Our primary objective will be to characterise the placebo response in TRD across various treatment modalities. Our exploratory aim will be to determine any demographic, clinical and methodological characteristics that contribute to it. Characterising and understanding what contributes to the placebo response in TRD are a crucial step towards the advancement of emerging treatments as well as potentially harnessing the placebo response for patients.

\section{METHODS AND ANALYSIS}

This protocol will be developed and reported in accordance with Preferred Reporting Items for Systematic Reviews and Meta-Analyses statement. ${ }^{16}$

\section{ELIGIBILITY CRITERIA}

\section{Participants and setting}

We will include RCTs that recruited patients with TRD of any gender and between 18 and 65 years old. TRD will be defined as patients with major depressive disorder (MDD) as defined by the Diagnostic and Statistical Manual of Mental Disorders III, IV, IV-R and V or International Classification of Diseases 9-10 that are currently in a depressive episode. ${ }^{17-19}$ Patients must have failed at least two trials of antidepressant medication within the current depressive episode with adequate dose and duration. Within class switches, for example, two selective serotonin reuptake inhibitors will be included as part of the TRD staging. ${ }^{20}$ Failed psychotherapy or brain stimulation trials will be included in the TRD staging. If a study reported that they included patients with two failed trials, but did not indicate whether this occurred within the current depressive episode, the study will be included as this is the most consistent definition of TRD. ${ }^{21-23}$ Patients from any setting (ie, inpatient or outpatient) will be included. Psychiatric comorbidity will be included, if MDD is the primary psychiatric disorder being treated.

We will exclude studies that recruited patients with bipolar depression, unless $15 \%$ or less of the patients randomised have bipolar depression, and patients diagnosed with primary psychotic illness or active substance use disorders. Patients with neurological disorders, physical comorbidities or medical conditions will only be excluded if these diagnoses are the primary diagnosis (eg, MDD in patients with diabetes or MDD in patients with multiple sclerosis). Studies with sample sizes less than 10 subjects $^{24}$ and studies that use a non-inert placebo will also be excluded.

\section{Interventions}

We will include pharmacological and somatic therapies that are included in the Maudsley Treatment Inventory. ${ }^{25}$ This inventory is derived from the Maudsley Prescribing Guidelines as well as other standardised guidelines for depression treatment. We will also include novel and repurposed agents that have multiple meta-analysis supporting their use.

For psychological agents, we will include those from the National Institute for Health and Care Excellence (NICE) guidelines, which include computerised or face-to-face cognitive behavioural therapy, behavioural activation, interpersonal therapy, manualised psychodynamic therapy, behavioural couples therapy, cognitive behavioural analysis system of psychotherapy or mindfulness-based cognitive therapy. ${ }^{22}$

\section{Comparator}

Trials include a placebo arm. Placebo will be defined as an inert oral medication, parental medication, sham device or sham therapy that does not include any theoretical active property to induce the proposed therapeutic effect. Wait-list or treatment as usual will not be considered a placebo group for therapy trials.

\section{Study designs and publication types}

We will only include parallel-arm, randomised, doubleblind placebo-controlled trials. We will include cross-over studies if they report outcomes before the cross-over. Trials must include randomisation to at least one placebo arm.

\section{Language and timeframe}

Studies to be included will be published in English or Portuguese. Attempts to translate other languages will also be made. Timeframe of included studies will be from the date of inception until 24th January 2020.

\section{INFORMATION SOURCE AND SEARCH STRATEGY}

The electronic databases MEDLINE and PsychINFO will be searched. Key terms, notable papers and citation lists will also be reviewed for additional studies. The following search terms will used in addition to mapping key terms: (depress* OR MDD OR major depress*) AND (resistan* OR refractor* OR non-respon* OR nonrespon* OR un-respon* OR unrespon* OR TRD OR fail* OR inadequate OR difficult OR intractable) AND (Placebo OR sham OR control OR controlled) AND (randomi* OR RCT) AND (treatment OR intervention OR trial).

\section{STUDY RECORDS}

\section{Study selection and data extraction}

Two authors will independently screen the abstracts and full texts to decide on their inclusion based on predefined inclusion criteria. Any discrepancies of inclusion or extraction will be discussed between the two authors, and a third author will resolve any further conflicts. Two 
authors will then extract data, which will include description of the interventions and control group, demographics, clinical data and quality assessment.

\section{OUTCOMES}

\section{Primary outcome}

The primary outcome will be 'placebo response' as measured by Hedges' $g$ effect size of the change in the primary outcome variable (ie, depression symptom rating scales) from baseline to primary endpoint. Where multiple outcomes are reported, the primary outcome for inclusion in analyses will be selected in a hierarchical fashion: the most preferable scale will be a clinician-rated assessment of depression severity (Hamilton Depression Rating Scale, Montgomery-asberg depression rating scale, Quick inventory of depression symptomatology, or validated subscales of these), followed by a patient-rated measure (Patient Health Questionaire-9, or Beck Depression Inventory). Where multiple endpoints are reported, this review will consider the acute endpoint as the primary endpoint. If the study only reports a delayed endpoint, this will be recorded and controlled for.

\section{Secondary outcomes}

1. Response rate measured by the percentage of patients who had a reduction of $\geq 50 \%$ of the total score on a standardised rating scale for depression.

2. Remission rates as measured by a standardised rating scale for depression.

\section{ASSESSMENT OF RISK BIAS}

We will use the Cochrane risk of bias tool. This tool assesses bias across five domains (selection, performance, attrition, reporting and others). A sensitivity analysis will assess the difference in statistical effects between studies with overall high and low risk of bias.

\section{DATA SYNTHESIS}

Qualitative data will be analysed, and sufficiently, homogenous studies will be aggregated based on similarity of patient characteristics, treatment modality and study design. We will conduct a pairwise meta-analysis within each modality. Placebo effect size will be determined by Hedges' $g$, which will be calculated based on reported means and SD from baseline and endpoint of each study. A random-effect model will be used to perform this calculation. When necessary, we will impute SD based on graphs, SE or CI provided in the published reports. The pooled effect size for each study will be calculated by the inverse variance of each study.

We will perform an explanatory analysis on factors affecting the placebo response using a univariate meta-regression. Several univariate meta-regressions will be performed for each treatment modality. Factors chosen will be dependent on data availability; however, examples include methodological factors, publication year, number of study sites, study setting, number of treatment arms, industry sponsorship, duration of study, number of days receiving placebo, augmentation versus monotherapy treatment strategy and study quality, as well as demographic factors (eg, age, gender and race/ethnicity) and clinical factors (eg, number of failed trials in the current episode, recurrence of illness, age of onset, baseline severity and effect size of the active group). For univariate meta-regression analyses, significant values will be considered as $\mathrm{p}<0.05$.

We will perform sensitivity analysis and cumulative regression and assess publication bias using Beggmodified funnel plot and Egger test. ${ }^{26}$ Heterogeneity will be evaluated with a $\mathrm{x}^{2}$ test.

\section{CONFIDENCE OF CUMULATIVE EVIDENCE}

The Grading of Recommendations, Assessment, Development and Evaluations approach will be used to the rank the quality of the evidence in making recommendations of what the placebo response in TRD is.

\section{PATIENT AND PUBLIC INVOLVEMENT}

TRD is a very significant public health concern. As there is no direct patient involvement in this study, we have decided to not include patients and public in the development in the protocol.

\section{DISCUSSION}

A placebo-controlled clinical trial is the gold standard for establishing efficacy of a proposed active treatment. While there is a well-established understanding of the placebo response in treatment-naive $\mathrm{MDD}$, there is not a clear understanding of the placebo response in TRD. Furthermore, the analyses of the placebo response in non-TRD focus almost entirely on the placebo response as it relates to oral medications. This has implications on the transferability of this knowledge to TRD as this patient population frequently uses somatic and novel treatments. The objective of this study is to better quantify the placebo response in TRD, its contributing factors and how it may differ between treatment modalities. This knowledge will help clinicians and researchers interpret past and future studies as well as improve the design and development of future trials. With an established placebo response, study designs such a noninferiority can be used with improved confidence. Lastly, this knowledge would have implications of how care can be delivered and improved for patients with TRD.

\section{Ethics and dissemination}

There is no research ethics board approval required. The dissemination plan is to publish results in a peer-reviewed academic journal.

\section{Author affiliations}

${ }^{1}$ Psychiatry, Centre for Addiction and Mental Health, Toronto, Ontario, Canada

${ }^{2}$ Psychiatry, University of Toronto, Toronto, Ontario, Canada 
${ }^{3}$ Department of Internal Medicine, Faculty of Medicine, University of Sao Paulo, Sao Paulo, Brazil

${ }^{4}$ Laboratory of Neurosciences (LIM-27), Instituto Nacional de Biomarcadores em Neuropsiquiatria (INBioN), Department and Institute of Psychiatry, Faculdade de Medicina, University of Sao Paulo, Sao Paulo, Brazil

${ }^{5}$ Psychiatry, University of California San Diego, San Diego, California, USA

Acknowledgements ARB receives grants from the National Council for Scientific and Technological Development (CNPQ, PQ-1B, to Dr. Brunoni), the Program of Academic Productivity (PIPA) of the University of São Paulo Medical School (to Dr. Brunoni) and from the São Paulo Research Foudation (Grant Number: 2018/108617). ARB is chief medical advisor of Flow ${ }^{\mathrm{TM}}$ and has a small equity of the company. The LIM-27 laboratory of neuroscience receives grants from the Associação Beneficente Alzira Denise Hertzog da Silva. LBR is supported by São Paulo ResearchFoundation (Grant Number: 2019/07256-7).

Contributors BDMJ made an exceptional contribution to the design/conception of the protocol, wrote the original version of the manuscript, gave final approval of the version published and has agreed to be accountable for all aspects of the work in ensuring that questions related to the accuracy or integrity of any part of the work are appropriately investigated and resolved. CW, LBR, MIH, ARB and ZD made an exceptional contribution to the design/conception of the protocol, made significant contributions to revisions of the manuscript, gave final approval of the version published and has agreed to be accountable for all aspects of the work in ensuring that questions related to the accuracy or integrity of any part of the work are appropriately investigated and resolved.

Funding The authors have not declared a specific grant for this research from any funding agency in the public, commercial or not-for-profit sectors.

Competing interests None declared.

Patient and public involvement Patients and/or the public were not involved in the design, conduct, reporting or dissemination plans of this research.

Patient consent for publication Not required.

Provenance and peer review Not commissioned; externally peer reviewed.

Open access This is an open access article distributed in accordance with the Creative Commons Attribution Non Commercial (CC BY-NC 4.0) license, which permits others to distribute, remix, adapt, build upon this work non-commercially, and license their derivative works on different terms, provided the original work is properly cited, appropriate credit is given, any changes made indicated, and the use is non-commercial. See: http://creativecommons.org/licenses/by-nc/4.0/.

ORCID iD

Brett D M Jones http://orcid.org/0000-0003-3248-1059

\section{REFERENCES}

1 Fountoulakis KN, Mclntyre RS, Carvalho AF. From randomized controlled trials of antidepressant drugs to the meta-analytic synthesis of evidence: methodological aspects lead to discrepant findings. Curr Neuropharmacol 2015;13:605-15.

2 Walsh BT, Seidman SN, Sysko R, et al. Placebo response in studies of major depression: variable, substantial, and growing. JAMA 2002;287:1840-7.

3 Khan A, Bhat A, Kolts R, et al. Why has the antidepressant-placebo difference in antidepressant clinical trials diminished over the past three decades? CNS Neurosci Ther 2010;16:217-26.

4 Sinyor $\mathrm{M}$, Levitt $\mathrm{AJ}$, Cheung $\mathrm{AH}$, et al. Does inclusion of a placebo arm influence response to active antidepressant treatment in randomized controlled trials? results from pooled and meta-analyses. J Clin Psychiatry 2010;71:270-9.

5 lovieno N, Papakostas GI. Does the presence of an open-label antidepressant treatment period influence study outcome in clinical trials examining augmentation/combination strategies in treatment partial responders/nonresponders with major depressive disorder? J Clin Psychiatry 2012;73:676-83.

6 Khan A, Khan SR, Walens G, et al. Frequency of positive studies among fixed and flexible dose antidepressant clinical trials: an analysis of the food and drug Administraton summary basis of approval reports. Neuropsychopharmacology 2003;28:552-7.

7 Landin R, DeBrota DJ, DeVries TA, et al. The impact of restrictive entry criterion during the placebo lead-in period. Biometrics 2000;56:271-8

8 Rutherford BR, Cooper TM, Persaud A, et al. Less is more in antidepressant clinical trials. J Clin Psychiatry 2013;74:703-15.

9 Whitlock ME, Woodward PW, Alexander RC. Is high placebo response really a problem in depression trials? A critical Re-analysis of depression studies. Innov Clin Neurosci 2019;16:12-17.

10 Targum SD, Cameron BR, Ferreira L, et al. Early score fluctuation and placebo response in a study of major depressive disorder. $J$ Psychiatr Res 2020;121:118-25.

11 Furukawa TA, Cipriani A, Atkinson LZ, et al. Placebo response rates in antidepressant trials: a systematic review of published and unpublished double-blind randomised controlled studies. Lancet Psychiatry 2016;3:1059-66.

12 Rush AJ, Warden D, Wisniewski SR, et al. STAR*D: revising conventional wisdom. CNS Drugs 2009;23:627-47.

13 Johnston KM, Powell LC, Anderson IM, et al. The burden of treatment-resistant depression: a systematic review of the economic and quality of life literature. J Affect Disord 2019;242:195-210.

14 Razza LB, Moffa AH, Moreno ML, et al. A systematic review and meta-analysis on placebo response to repetitive transcranial magnetic stimulation for depression trials. Prog Neuropsychopharmacol Biol Psychiatry 2018;81:105-13.

15 Brunoni AR, Lopes M, Kaptchuk TJ, et al. Placebo response of nonpharmacological and pharmacological trials in major depression: a systematic review and meta-analysis. PLoS One 2009;4:e4824.

16 Shamseer L, Moher D, Clarke M, et al. Preferred reporting items for systematic review and meta-analysis protocols (PRISMA-P) 2015 elaboration and explanation. BMJ 2015;349:g7647.

$17 \mathrm{WHO}$. The ICD-10 classification of mental and behavioural disorders clinical descriptions and diagnostic guidelines. Available: http://www. who.int/classifications/icd/en/bluebook.pdf

18 First M, Gibbon M, Spitzer R. User guide for the structural clinical interview for DSM-IV axis I disorders. Washington, DC: American Psychiatric Association, 1996.

19 American Psychiatric Association. Diagnostic and statistical manual of mental disorders. 4th edn. Washington, DC: American Psychiatric Association, 1994.

20 Papakostas GI, Fava M, Thase ME. Treatment of SSRI-resistant depression: a meta-analysis comparing within- versus across-class switches. Biol Psychiatry 2008;63:699-704

21 Souery D, Amsterdam J, de Montigny C, et al. Treatment resistant depression: methodological overview and operational criteria. Eur Neuropsychopharmacol 1999;9:83-91.

22 NICE. Depression in adults (update) depression: the treatment and management of depression in adults national clinical practice guideline number $X$ national collaborating centre for mental health commissioned by the National Institute for health and clinical Exc, 2007.

23 Anderson IM, Ferrier IN, Baldwin RC, et al. Evidence-Based guidelines for treating depressive disorders with antidepressants: a revision of the 2000 British association for psychopharmacology guidelines. J Psychopharmacol 2008;22:343-96.

24 Bandelow B, Reitt M, Röver C, et al. Efficacy of treatments for anxiety disorders: a meta-analysis. Int Clin Psychopharmacol 2015;30:183-92.

25 Fekadu A, Wooderson SC, Markopoulou K, et al. The Maudsley staging method for treatment-resistant depression: prediction of longer-term outcome and persistence of symptoms. J Clin Psychiatry 2009;70:952-7.

26 Egger M, Davey Smith G, Schneider M, et al. Bias in meta-analysis detected by a simple, graphical test. BMJ 1997;315:629-34. 